\title{
The time to begin plans for COVID-19 eradication is now
}

\author{
T Jacob Johna, Dhanya Dharmapalan ${ }^{b}$ \\ a PhD (Virology), FRCP (Paediatrics), Former Professor \& Head, Department of Clinical Virology, Christian \\ Medical College, Vellore, India \\ ${ }^{\mathrm{b}}$ MBBS, MD, Consultant, Paediatric Infectious Diseases at Apollo Hospitals, Navi Mumbai, India
}

\begin{abstract}
After the world recovers from the pandemic of SARS-CoV-2, it is most likely to stabilise as endemic and seasonal, deserving/demanding control efforts perpetually in all countries, unless it can be eradicated. The risk of mortality is high among those above 65 years and those with chronic "lifestyle" diseases. Endemic circulation will, therefore, take a heavy toll on life annually. Eradication is an extreme form of control, eliminating the disease permanently and globally. Effective vaccines are expected in the near future. As the pandemic abates, herd immunity will be very high, enabling early eradication by additional build-up of a vaccine-induced herd immunity. Public memory of the pandemic will be fresh, which will assist in social mobilisation and fund raising towards eradication. If time is lost, the infection is likely to become non-eradicable as domestic/farmed animals may become fresh reservoirs. Resolve to eradicate and designing its road-map must be made at the earliest.
\end{abstract}

Key words: COVID-19, pandemics, vaccines, global health

"We cannot solve our problems with the same thinking we used when we created them." (Einstein)

\section{The COVID-19 pandemic, a veritable nightmare}

The pandemic of the Coronavirus disease2019 (COVID-19), caused by an infection with Severe Acute Respiratory Syndrome Coronavirus type 2 (SARS-CoV-2), has sowed disease, death, and devastation in its path across all countries of the world. By the end of June 2020, it has caused over 9.4 million cases and over 480,000 deaths

November 2020. Christian Journal for Global Health 7(4) globally. ${ }^{1}$ Risks of severe disease and death are high in those above 65 years and those with chronic non-communicable diseases (co-morbidities). ${ }^{2}$ Children, adolescents, and young healthy adults tend to develop asymptomatic infection or only mild symptoms and are at low risk of death.

The mode and speed of the spread of SARSCoV-2 resembles that of influenza viruses. The 1918 influenza pandemic (causative virus identified by paleo-virology as A H1N1) had three successive waves in March, SeptemberNovember, and early 1919. ${ }^{3}$ H1N1 virus was replaced by $\mathrm{H} 2 \mathrm{~N} 2$ when it became a pandemic in 1957, which was then replaced by the H3N2 
pandemic virus of $1968,{ }^{4}$ but the 2009 pandemic H1N1 virus did not replace H3N2, and since then, both H1N1 and H3N2 are endemic globally. Each pandemic virus became globally endemic unless replaced by another influenza virus, and SARSCoV-2 will most likely emerge as globally endemic. $^{5}$ We have no reason to expect a less virulent beta coronavirus to emerge and replace this highly virulent pandemic virus.

Two other coronaviruses have emerged as zoonosis first and then human-to-human transmission continued: the SARS-CoV-1 and the MERS-CoV, causing severe acute respiratory syndrome (SARS) and the Middle-Eastern respiratory syndrome, respectively. The former did become a pandemic involving 27 countries, resulting in 8,096 cases and 774 deaths. ${ }^{6}$ By diligently case diagnosing, contact tracing, and quarantining, SARS was eradicated within one year without a vaccine or anti-viral drug.

This approach failed with SARS-CoV-2 because of greater transmission efficiency and the fact that infected asymptomatic and presymptomatic (during incubation period) individuals transmitted infection, whereas SARS virus was transmitted only after a fever developed, allowing case detection and breaking of transmission chains. Fortunately, it has not reappeared as zoonosis in the country of origin as precautions were taken.

MERS-CoV has appeared several times as a zoonosis, often resulting in limited person-toperson transmission. Further spread could be interrupted so that it has neither persisted as anthroponosis nor became a pandemic. However, when imported into the Korean Republic, it caused a major epidemic, but once detected and when nonpharmacological interventions were applied, it could be eliminated. ${ }^{7}$ SARS-CoV-2 is a highly efficient person-to-person transmitter and, within a span of 4 months from first recognition in China, it has reached all United Nations member countries. It is now fully entrenched globally as anthroponosis and it would be naive and foolhardy to expect that its transmission could be interrupted everywhere using non-pharmacological interventions.

To presume it will die out will turn out to be a huge risk if it does not. The world will be safer if we presume the opposite - that it will become pan-endemic when herd immunity grows sufficiently high. ${ }^{5}$ In the medium term, as the disease remains endemic with annual seasonal prevalence, senior citizens and those with comorbidities will have to live in fear of infection and its consequences, unless effective anti-viral therapy is developed or at least one safe and effective vaccine becomes available, accessible, and affordable even in low income countries, or unless the disease is eradicated.

\section{Can COVID-19 be eradicated?}

Disease eradication is defined as permanent reduction to zero of the worldwide incidence of the causative infection, with deliberate efforts. ${ }^{8}$ For COVID-19 eradication, the coronavirus transmission must be interrupted globally.

Three biomedical criteria determine if a disease can be eradicated: i) availability of vaccine for primary prevention; ii) availability of a diagnostic tool to reliably detect infection; iii) absence of extra-human reservoir of the agent. ${ }^{8}$

Although SARS-CoV-2 only recently jumped species from bats to humans as a zoonosis, it is now an anthroponosis, meaning efficiently spreading person-to-person, hence theoretically eradicable. ${ }^{8}$ The presumptive parent virus in the bat is a millennia old. Its emergence as an emerging anthroponosis of zoonotic origin had not happened before 2019, so we have to presume it is a one-off event. As an anthroponosis, it is eradicable, in spite of its original reservoir remaining in nature. However, constant vigilance and escalated surveillance for any new zoonotic to human introduction is needed.

If such re-introductions recur, eradication may have setbacks, but we will know if reintroduction, if any, since surveillance will be of eradication quality. Thus, if we fear reintroduction of either the same or another coronavirus capable of easy/rapid spread, we must eradicate the current 
one, instead of remaining unprepared for such eventualities.

Diagnostic tests like reverse transcription polymerase chain reaction (RT-PCR) and antibody tests are already available. SARS-CoV-2 is excreted in the faeces and found in sewage. Environmental surveillance of sewage for SARSCoV-2 can, therefore, be used to detect infection if during the eradication programme it silently reemerges in the community. This is a lesson we have learned from environmental surveillance of the polio eradication programme.

The critical intervention tools are vaccines to build herd immunity sufficient to interrupt transmission. Currently several vaccine candidates are in the pipeline. We expect more than one vaccine to be on the market by 2021. Since infection and transmission are based on upper respiratory mucosal infection, the best vaccine that offers upper respiratory mucosal protection should be used for eradication. Since basic reproduction number Ro is below 4, theoretically the herd immunity threshold for interruption of transmission would be about $75 \% .^{9}$ Vaccination coverage has to top up the existing herd immunity due to natural infection. Vaccine efficacy will determine the coverage needed: the higher the efficacy, the lower the coverage needed.

\section{Why think of eradication when the pandemic is still on?}

Wisdom calls for early eradication of the virus without providing it time and opportunity to better adapt to human hosts and alter behaviour, antigenicity, or virulence.

We must pre-empt the virus from adapting itself to non-human vertebrates, potentially allowing them to become non-human reservoirs. If that happens, COVID-19 will become theoretically non-eradicable. Already canines, felines, and fur animals (mink and fox) are found to be susceptible to infection-we must not allow time for virus adaptation to any domestic, farm, or wild animals. As mink-to-human transmission has already occurred on limited occasions, no doubt there will be strict infection control in mink farms, but leaks are always possible. ${ }^{10}$ Although such infections have been stray instances of reverse zoonosis (human-to-vertebrate transmission), the natural extension of transmissibility is continued enzootic transmission.

Non-pharmacological deterrents of transmission require social distancing. Humans need social interactions from the cradle to the deathbed. Economic reconstruction and administrative, health management, recreational, educational, and religious activities require social interactions. However, social interactions will promote virus transmission when it is endemic, a potential catch-22.

Most important, we must galvanize the world to prepare for eradication while the memory of the nightmare of the pandemic remains fresh in the public's mind and when public cooperation can readily be solicited. Technically, the best opportunity to eradicate the coronavirus will be in the near-term, when herd immunity will be extremely high as the pandemic peaks and evolves as pan-endemic.

\section{What will it take to develop a global agenda for eradication?}

Eradication becomes a global agenda only when the World Health Assembly passes a resolution to commit the World Health Organisation and its member nations to join in partnership to achieve this goal. For the reasons argued above, eradication must be achieved in the shortest possible time-ideally as short as five years or perhaps a little longer, according to practicality and our collective wisdom. Eradication has to be formally certified, which will take one or two more years.

An innovative immunisation platform will have to be imaginatively created as the existing ones, such as the Expanded Programme on Immunisation, will be insufficient for eradication. The majority of infections are asymptomatic, hence invisible and silent. Children must be vaccinated in spite of low risk of disease; adolescents and adults, young and old, must be vaccinated although individually they may feel no 
reason for it. Herd immunity must be rapidly built up through high vaccination coverage. The pandemic has taught us a new way of looking at human health problems - and that innovative solutions must be developed.

Eradication, as an inevitably gargantuan project, will require a huge commitment of human and financial resources across the globe. Implementation will require innovative financial design and must be faster than our past performances addressing global public health problems, including polio eradication. Scepticism from strong quarters is to be expected and must be countered through persuasive thought leadership and a concerted global educational campaign.

The interventions for effective control of the pandemic or country-level epidemics, and for eradication, should not be conceived of as sequential but as simultaneous or overlapping as necessary. We should be ready to implement the eradication programme as soon as a primary prevention tool becomes available. All the deliberations needed and the resolve and resolutions commensurate with the enormity of the challenges should be ready to be in fast-forward gear.

"Everything is created twice - once in the mind and then in reality" (Robin Sharma). Let us get the first part done by the time reality opens its door for us.

\section{References}

1. World Health Organisation. Coronavirus disease (COVID-19) situation report-158, 2020 June 26 [cited 2020 June 27]. Available from: https://www.who.int/docs/defaultsource/coronaviruse/situation-reports/20200626covid-19-sitrep-158.pdf?sfvrsn=1d1aae8a_2

2. CDC COVID-19 Response Team. Severe outcomes among patients with coronavirus disease 2019 (COVID-19) - United States, February.12March 16, 2020. MMWR Morb Mortal Wkly Rep 2020;69(12):343-6. http://dx.doi.org/10.15585/mmwr.mm6912e2
3. Taubenberger JK, Morens DM. 1918 Influenza: the mother of all pandemics. Emerg Infect Dis. 2006;12(1):15-22.

http://dx.doi.org/10.3201/eid1201.050979

4. Dharmapalan D. Influenza [published online ahead of print, 2020 Feb 11]. Indian J Pediatr. 2020;1-5. http://dx.doi.org/10.1007/s12098-02003214-1

5. John TJ. Will coronavirus pandemic eventually evolve as pan-endemic? Current Science. 2020;118(6):855-6. Available from: https://www.scopus.com/record/display.uri?eid=2 $\underline{-\mathrm{s} 2.0-}$

85085976260\&origin $=$ inward\&txGid $=$ e1083edc3 cb277e3b8a1bcdfbcca8e84

6. Cherry JD. The chronology of the 20022003 SARS mini pandemic. Paediatr Respir Rev. 2004;5(4):262-9. http://dx.doi.org/10.1016/j.prrv.2004.07.0 $\underline{09}$

7. Al-Omari A, Rabaan AA, Salih S, AlTawfiq JA, Memish ZA. MERS coronavirus outbreak: implications for emerging viral infections. Diagn Microbiol Infect Dis. 2019;93(3):265285.

http://dx.doi.org/10.1016/j.diagmicrobio. 2018.10.011

8. Dowdle WR. The principles of disease elimination and eradication. Morb Mortal Wkly Rep 1999;48(SU01):23-7. Available from: https://www.cdc.gov/mmwr/preview/mmwrhtml/s $\underline{\mathrm{u} 48 \mathrm{a} 7 . \mathrm{htm}}$

9. Liu Y, Gayle AA, Wilder-Smith A, Rocklöv J. The reproductive number of COVID-19 is higher compared to SARS coronavirus. J Travel Med; 2020;27(2):1-4. http://dx.doi.org/10.1093/jtm/taaa021

10. Mink infected two humans with coronavirus: Dutch Government. Health. 2020 May 25[cited 2020 May 27]. Available from: https://www.usnews.com/news/world/articles/202 0-05-25/dutch-government-second-case-of-minktransmitting-coronavirus-to-human 
Submitted 11 June 2020, accepted 13 Aug 2020, published 9 Nov 2020

Competing Interests: None declared.

Correspondence: Dhanya Dharmapalan, Apollo Hospitals, Navi Mumbai, India.

drdhanyaroshan@gmail.com

Cite this article as: John JT, Dharmapalan D. The time to begin plans for COVID-19 eradication is now. Christ J Global Health. November 2020; 7(4):47-51 https://doi.org/10.15566/cigh.v7i4.481

(C) Authors. This is an open-access article distributed under the terms of the Creative Commons Attribution License, which permits unrestricted use, distribution, and reproduction in any medium, provided the original author and source are properly cited. To view a copy of the license, visit http://creativecommons.org/licenses/by/4.0/

www.cjgh.org 